\title{
Burn injury during long-term oxygen therapy in Denmark and Sweden: the potential role of smoking
}

\author{
Hanan A Tanash' \\ Thomas Ringbaek ${ }^{2}$ \\ Fredrik Huss ${ }^{3,4}$ \\ Magnus Ekström' \\ 'Department of Respiratory Medicine, \\ Skåne University Hospital, Lund \\ University, Lund, Sweden; \\ ${ }^{2}$ Respiratory Department, \\ Hvidovre Hospital, Copenhagen, \\ Denmark; ${ }^{3}$ Department of Surgical \\ Sciences, Plastic Surgery, ${ }^{4}$ Department \\ of Plastic and Maxillofacial Surgery, \\ Burn Center, Uppsala University \\ Hospital, Uppsala, Sweden
}

Correspondence: Hanan A Tanash Department of Respiratory Medicine, Skåne University Hospital, Inga Marie Nilssons Gata 46, S-205 02 Malmö, Sweden

Email hanan.tanash@med.lu.se
This article was published in the following Dove Press journal: International Journal of COPD

5 January 2017

Number of times this article has been viewed

Background: Long-term oxygen therapy (LTOT) increases life expectancy in patients with COPD and severe hypoxemia. Smoking is the main cause of burn injury during LTOT. Policy regarding smoking while on LTOT varies between countries. In this study, we compare the incidence of burn injury that required contact with a health care specialist, between Sweden (a country with a strict policy regarding smoking while on LTOT) and Denmark (a country with less strict smoking policy).

Methods: This was a population-based, cohort study of patients initiating LTOT due to any cause in Sweden and Denmark. Data on diagnoses, external causes, and procedures were obtained from the Swedish and Danish National Patient Registers for inpatient and outpatient care. Patients were followed from January 1, 2000, until the first of the following: LTOT withdrawal, death, or study end (December 31, 2009). The primary end point was burn injury during LTOT.

Results: A total of 23,741 patients received LTOT in Denmark and 7,754 patients in Sweden. Most patients started LTOT due to COPD, both in Sweden (74\%) and in Denmark (62\%). The rate of burn injury while on LTOT was higher in Denmark than in Sweden; 170 (95\% confidence interval [CI], 126-225) vs 85 (95\% CI, 44-148) per 100,000 person-years; rate ratio 2.0 (95\% CI, 1.0-4.1). The risk remained higher after adjustment for gender, age, and diagnosis in multivariate Cox regression, hazard ratio 1.8 (95\% CI, 1.0-3.5). Thirty-day mortality after burn injury was $8 \%$ in both countries.

Conclusion: Compared to Sweden, the rate of burn injury was twice as high in Denmark where smoking is not a contraindication for prescribing LTOT.

Keywords: burn injury, COPD, long-term oxygen therapy, smoking

\section{Introduction}

Long-term oxygen therapy (LTOT) improves the survival time for patients with COPD and severe hypoxemia but may engender an increased risk of fire accidents and burn injury. ${ }^{1}$ Oxygen accelerates fire through increasing the risk of starting a fire and to make it more violent. Smoking is the main cause of burn injury during LTOT. ${ }^{2}$ Patients on LTOT are warned that they must avoid contact with sources of sparks and open flames, such as smoking cigarettes, candles flames, and open fire.

The first, population-based, longitudinal study of burn injury during LTOT recently reported a low incidence of 61 per 100,000 person-years in Sweden, where only 2\% of patients starting LTOT were active smokers. ${ }^{3}$

Prescribing oxygen to patients who continue to smoke is controversial, and policy regarding smoking while on LTOT varies among countries and settings. The prevalence of active smokers while on LTOT has, however, been reported to be significantly high 
in other Western countries, including 21\% in Denmark. ${ }^{4,5}$ Smoking is an absolute contraindication for LTOT in Sweden but not in Denmark, which may explain the difference in the prevalence of smokers on LTOT. It is not known whether a strict policy regarding smoking during LTOT contributes to a reduction in the incidence of burn injuries.

Denmark and Sweden offer unique opportunities to study adverse events during LTOT due to the existence of population-based prospective longitudinal registers of LTOT patients, with complete follow-up through cross-linkage with national health care and mortality registers.

The primary aim of this study was to examine whether the incidence of burn injuries during LTOT, which required contact with a health care specialist, is higher in Denmark than in Sweden. Second, we aimed to explore the clinical factors associated with the increased risk of burn injury.

\section{Methods}

\section{Study design and population}

This was a prospective, population-based, consecutive cohort study. Inclusion criteria were people commencing with LTOT between January 1, 2000, and December 31, 2009, in the Swedish Register of Respiratory Failure (Swedevox) and the Danish Oxygen Register. Exclusion criterion was cancer as primary cause for the initiation of LTOT. Patients were followed prospectively from January 1, 2000, until the first of LTOT withdrawal, death, or study end (December 31, 2009). The primary end point was burn injury during LTOT.

\section{The Swedish Register and the Danish Oxygen Register}

Swedevox covers $\sim 85 \%$ of all adults on LTOT in Sweden (9.1 million inhabitants) since January 1, 1987. The Danish Oxygen Register was established in November 1994 and covers $>99 \%$ of the Danish population (5.4 million inhabitants). The registers have been detailed elsewhere., The Swedish and Danish indications for LTOT are in line with international guidelines. ${ }^{8}$ Contraindications that have been applied since 1987 in Sweden are continued smoking and contact with gas appliances or open fire. In Denmark, smoking is not an absolute contraindication for LTOT. LTOT may still be prescribed to patients who continue to smoke, given that the patient understands the importance of avoiding contact between the supplemental oxygen and any source of fire.

\section{Assessments}

Data on covariates were obtained from the Swedish and Danish registers, regarding age, gender, and the physician-diagnosed disease, which was the main reason for initiating LTOT. Smoking status at baseline was available only for Swedish patients.

Data on diagnoses, external causes, and procedures coded according to the 10th revision of the WHO International Classification of Diseases (ICD) were obtained from the Swedish and Danish National Patient Registers for inpatient and outpatient care. ${ }^{9-11}$ The Swedish register covers $>99 \%$ of all hospitalizations since 1987 and $\sim 80 \%$ of all hospitalbased outpatient care since 2001 in Sweden. ${ }^{10}$ The Danish Register covers inpatient care since 1977 and all outpatient care and emergency contacts in Denmark since 1995. ${ }^{11}$ Vital status was obtained from the National Register of Causes of Death of each country.

\section{Definition of burn injury}

Burn injury during LTOT was assessed via the same two-step process in both Denmark and Sweden. First, all ICD codes in the National Patient Register and Causes of Death Register of the countries were screened and categorized as possible burn injury ( $\mathrm{n}=1,463$ patients), using the definition as previously published. ${ }^{3}$ Second, the study database was examined by a senior plastic surgeon specialized in burn care $(\mathrm{FH})$, who manually evaluated all ICD-codes from the health care contacts with a diagnosis of possible burn injury $(n=195)$. Each event was categorized either as burn injury $(n=61)$ subcategorized as skin or inhalational injury, or no burn injury. The categorization was cross-validated by a second author (ME). Uncertain codes were categorized through consensus discussion (FH and ME), and if still unclear, they were categorized as burn injuries. The assessment and categorization were blinded to all patient covariates. Death related to burn injury was defined as any death occurring within 30 days after a burn injury.

\section{Ethical considerations}

The study was approved in Sweden by the Lund University Research Ethics Committee (157/2007 and 350/2008), the National Board of Health and Welfare, and the Data Inspection Board. In Denmark, the study was approved by the Danish Regional Ethical Committee and Data Inspection Board. According to national legislations, individual consent was not required as the analysis included only already collected registry data.

\section{Statistical analysis}

Baseline characteristics were tabulated using descriptive statistics.

The rate of burn injuries was calculated as the number of first burn injuries divided by the time at risk defined as 
person-years from starting LTOT until date of first burn injury diagnosis censored at LTOT withdrawal, death, or study end (December 31, 2009), whichever occurred first. Stratified analysis of the rate of burn injury was performed according to the possible risk factors at baseline: age, gender, and underlying diseases.

The relative risk of burn injury in Denmark compared with Sweden was analyzed using Cox regression adjusted for age, gender, and underlying diseases. We calculated 95\% confidence intervals (CIs) for all estimates. Rate ratio (RR) is computed as the ratio of the incidence rate in the Danish register divided by the incidence rate in given Swedevox.

Statistical analyses were performed with the Statistical Package for the Social Sciences (SPSS), version 22.0 (IMB Corporation, Armonk, NY, USA).

\section{Results}

\section{Patients}

During the study period, a total of 23,741 patients started LTOT in Denmark and 7,754 patients in Sweden. Patient characteristics at commencement of LTOT are shown in Table 1. Swedish patients were significantly older than Danish patients and had a longer follow-up time $(P<0.001)$. Most patients started LTOT due to COPD, both in Sweden (74\%) and Denmark (62\%). Smoking status at baseline was available for 7,373 (95\%) patients in Swedevox, of whom 6,262 (85\%) were ex-smokers, 980 (13\%) never-smokers, and $131(2 \%)$ current-smokers.

\section{Burn injuries during LTOT}

The mean rates of burn injury, stratified by age, gender and underlying disease, are shown in Table 2. The rate of burn injury was higher in Denmark than in Sweden; 170 (95\% CI, 126-225) vs 85 (95\% CI, 44-148) burn injuries per 100,000 person-years on LTOT; RR $2.0(95 \%$ CI, 1.0-4.1).

Table I Characteristics of 31,495 patients starting long-term oxygen therapy in Sweden and Denmark 2000-2009

\begin{tabular}{lll}
\hline Characteristic & $\begin{array}{l}\text { Denmark, } \\
\mathbf{N}=\mathbf{2 3 , 7 4 I}\end{array}$ & $\begin{array}{l}\text { Sweden, } \\
\mathbf{N}=\mathbf{7 , 7 5 4}\end{array}$ \\
\hline Men & $10,147(43)$ & $3,592(46)$ \\
$\begin{array}{l}\text { Mean age at start (range), years } \\
\text { Main diagnosis }\end{array}$ & $73(18-104)$ & $74(25-98)^{*}$ \\
COPD & $14,775(62)$ & $5,767(74)$ \\
Pulmonary fibrosis & $973(4)$ & $1,277(17)$ \\
Others & $7,993(34)$ & $710(9)$ \\
Median follow-up (IQR), years & $0.54(\mathrm{I} .5)$ & $\mathrm{I} .2(2.1)^{*}$ \\
Deaths during follow-up & $19,717(83)$ & $5,882(76)$ \\
\hline
\end{tabular}

Notes: Data presented as $n(\%)$ unless otherwise specified. $* P<0.00$ I.
In univariate analysis, lower age and LTOT in Denmark were associated with a higher risk of burn injury (Table 3). After adjustment for gender, age, and diagnosis in multivariate Cox regression, the risk of burn injury was higher in Denmark than in Sweden, hazard ratio (HR) 1.8 (95\% CI, 1.0-3.5, Table 3). The increased rate of burn injuries in Denmark was driven by a higher risk taken by Danish men. Danish men had a 6.6 times higher risk of burn injury compared with Swedish men; HR 6.6 (95\% CI, 1.6-27.6). The risk for women was similar in the two countries; HR 1.0 (95\% CI, 0.5-2.2).

Among the 61 patients with burn injuries, the 30-day mortality was $8 \%$ in both Sweden (one death) and Denmark (four deaths).

\section{Discussion}

\section{Main findings}

This prospective, population-based, cohort study examined whether the policy regarding smoking while on LTOT affects the incidence of burn injuries by comparing Sweden (strict policy) with Denmark (less strict smoking policy). Our results showed that the rate of burn injury was almost twice as high in Denmark as in Sweden. The high risk of burn injury in Denmark was driven by the increased risk among men; the risk for men vs women was more than six times higher in Denmark compared with in Sweden.

\section{Mechanisms}

The lower incidence of burn injury in Sweden compared with Denmark could be explained by the fact that active smoking is an established contraindication for LTOT in Sweden. For most smokers, LTOT is withheld, or terminated, after reasonable harm reduction techniques have failed. In this study, only $2 \%$ of Swedish patients were smokers. In the Danish register, smoking is stated as a contraindication for LTOT. However, LTOT can still be prescribed in the setting of severe hypoxemia and moderate smoking if the patient is deemed to have a sufficient understanding of the fact that it is imperative that oxygen must be kept away from fire. The number of active smokers on LTOT was estimated to be higher than 20\%-25\% in Denmark. ${ }^{4,5}$

Higher age when LTOT is commenced is associated with lower risk of burn injury in both Denmark and Sweden. This is in line with studies from the USA, where the mean age of patients on LTOT with burn injuries, was 62-64 years, ie, significantly younger than the LTOT patients in general. ${ }^{1,12,13}$ A previous study has shown that at least $21 \%$ of Danish COPD patients on LTOT were still smoking, and the smokers were on average 2.3 years younger than the nonsmokers. ${ }^{5}$ 
Table 2 Burn injuries while on LTOT in 31,495 patients starting therapy in Sweden and Denmark 2000-2009

\begin{tabular}{|c|c|c|c|c|}
\hline \multirow[t]{2}{*}{ Patient group } & \multicolumn{2}{|l|}{ Denmark } & \multicolumn{2}{|l|}{ Sweden } \\
\hline & n/person-years & Rate $(95 \% \mathrm{Cl})$ & n/person-years & Rate $(95 \% \mathrm{Cl})$ \\
\hline Overall & $49 / 28,798$ & $170(126-225)$ & $12 / 14,146$ & $85(44-148)$ \\
\hline Men & $26 / 11,599$ & $224(146-328)$ & $2 / 5,789$ & $35(4-125)$ \\
\hline Women & $23 / 17,199$ & $134(85-200)$ & $10 / 8,357$ & $120(57-220)$ \\
\hline \multicolumn{5}{|l|}{ Age at start } \\
\hline$>70$ years & $21 / 15,982$ & $|3|(8 \mid-200)$ & $5 / 8,244$ & $61(20-142)$ \\
\hline$\leq 70$ years & $28 / 12,815$ & $218(145-316)$ & $7 / 5,901$ & $119(48-244)$ \\
\hline \multicolumn{5}{|l|}{ Main diagnoses } \\
\hline COPD & $36 / 20,716$ & I 74 (I22-24I) & $9 / 11,036$ & $82(37-155)$ \\
\hline Others & $13 / 8,082$ & $161(86-275)$ & $3 / 3,110$ & $96(20-282)$ \\
\hline Ever-smokers & No data & No data & $10 / 12,066$ & $83(40-152)$ \\
\hline Never-smokers & No data & No data & $2 / 1,528$ & $|3|(\mid 6-472)$ \\
\hline
\end{tabular}

Notes: n, number of burn injuries; person-years, total person-years of LTOT; Rate $(95 \% \mathrm{Cl})$, rate per 100,000 person-years $(95 \%$ confidence interval).

Abbreviations: LTOT, long-term oxygen therapy; $\mathrm{Cl}$, confidence interval.

Thus, if we expect that Danish patients with burn injuries were predominantly smokers, this could be one explanation of the lower age among patients with burn injuries.

COPD was the main underlying cause of LTOT in both countries; $74 \%$ of patients in Sweden and 62\% in Denmark. There was a difference in the prevalence of other causes between the countries. The risk of burn injury in Denmark was higher for both COPD and other diagnoses, and the findings were similar after adjusting for underlying diagnosis (COPD/other) in the multivariate model. Our interpretation is that the difference in underlying causes of LTOT between countries did not influence the study findings.

The 30-day mortality rate after burn injury was $8 \%$ in both countries. This is lower compared with the study by Carlos et $\mathrm{l}^{12}(\mathrm{n}=55)$, which reported that $14.5 \%$ of patients died within a mean of 10 days (range, 1-53 days) after discharge from a burn unit for smoking-related, home oxygen injury. The lower mortality rate in the present study could be explained by a less selected population and by capturing less severe burn injuries using national hospitalization and outpatient data.

\section{Implications}

The present findings suggest that the risk of burn injury is increased in a setting where smoking while on LTOT is

Table 3 Univariate and multivariate Cox regression of the risk of burn injury while on LTOT

\begin{tabular}{lll}
\hline Factor & $\begin{array}{l}\text { Univariate model, } \\
\text { hazard ratio }(\mathbf{9 5 \%} \mathrm{CI})\end{array}$ & $\begin{array}{l}\text { Multivariate model, } \\
\text { hazard ratio }(\mathbf{9 5 \%} \mathbf{~ C I})\end{array}$ \\
\hline Denmark vs Sweden & $\mathrm{I} .9(\mathrm{I} .0-3.7)$ & $\mathrm{I} .8(\mathrm{I} .0-3.5)$ \\
Men vs women & $\mathrm{I} .2(0.7-2.0)$ & $\mathrm{I} .2(0.7-2.0)$ \\
Age at start & $0.97(0.95-0.99)$ & $0.97(0.95-0.99)$ \\
COPD vs other & $1.02(0.6-1.8)$ & $\mathrm{I} .2(0.7-2.3)$ \\
diagnosis & & \\
\hline
\end{tabular}

Abbreviations: LTOT, long-term oxygen therapy; $\mathrm{Cl}$, confidence interval. more prevalent, and support that active smoking should be considered as a contraindication for LTOT. The increased burn rate in Denmark (high smoking setting) compared with Sweden (low smoking setting) could translate into an even markedly higher risk in relation to smoking, as the likely prevalence of active smoking while on LTOT was $<5 \%$ in Sweden and $\sim 21 \%$ in Denmark.

The clinical effect of LTOT in smokers has been a subject of debate. Smokers with hypoxemia who use oxygen for 15-24 hours daily may have an increased risk of burn injury but probably also improved survival as a consequence of LTOT. Fifty percent of patients in the randomized NOTT trial were smokers, but there are no published data on whether smoking status had an impact on survival. ${ }^{14,15}$ Although randomized trials would show a positive effect of LTOT for smokers with hypoxemia, many will probably still be cautious in recommending LTOT for smokers because of the likely increased risk of fire and burn injury.

In agreement with previous studies, we suggest that there is a need to update the guidelines regarding smoking during LTOT. ${ }^{2,12,16}$ Efforts to cease smoking should be optimized. Despite the fact that there is a significant subset of COPD patients who continue to smoke, only few agencies and respiratory societies consider smoking an exclusion or absolute contraindication for oxygen therapy. ${ }^{16}$ Guidelines are needed to encourage clinicians to regularly check smoking status of their LTOT patients, repeatedly and continuously inform patients about the dangers of smoking while on LTOT, and reassess indications for LTOT.

\section{Strengths and limitations}

To our knowledge, the current study is the first study that compares the incidence of burn injury between two countries with different policies regarding smoking during LTOT. 
This study has a binational, longitudinal, population-based design with high coverage, including all hospitalizations since 1987 and data from the Causes of Death Registers. Burn injuries requiring medical care were identified using a sensitive screening of all diagnoses, external causes, procedural codes, and causes of death and were ascertained by a specialist in burn care and cross-validated by a second author. The categorization of burn injuries was blinded for all patient covariates.

There were several possible limitations. The major limitation was that smoking status was not available for Danish patients at baseline and was approximated from previous Danish studies that published between 1994 and 2001. The incidence of burn injury pertains to events causing hospitalization or specialist consultation. We had limited data on the characteristics and circumstances surrounding the burn injuries, of which some might have been unrelated to the LTOT. Data on the patients' living conditions were limited, as were the data on the extent and severity of burn injuries.

\section{Conclusion}

The risk of burn injury seems to be relatively low overall but increased in a setting where smoking is not a contraindication for LTOT. There is a need to develop the guidelines regarding smoking during LTOT.

\section{Acknowledgments}

We thank all the staff who registered the data, and all the doctors and nurses who cared for the patients. ME was supported by unrestricted grants from the Swedish Society of Medicine, the Swedish Respiratory Society, the Scientific Committee of Blekinge County Council, the Wera and Emil Cornell Foundation, and the Swedish Heart-Lung Foundation. FH was supported by unrestricted grants from the Swedish Society of Medicine and local funds from Uppsala County Council (ALF project).

\section{Disclosure}

The authors report no conflicts of interest in this work. The authors alone are responsible for the content and writing of the paper.

International Journal of COPD

\section{Publish your work in this journal}

The International Journal of COPD is an international, peer-reviewed journal of therapeutics and pharmacology focusing on concise rapid reporting of clinical studies and reviews in COPD. Special focus is given to the pathophysiological processes underlying the disease, intervention programs, patient focused education, and self management protocols.

\section{References}

1. Assimacopoulos EM, Liao J, Heard JP, Kluesner KM, Wilson J, Wibbenmeyer LA. The national incidence and resource utilization of burn injuries sustained while smoking on home oxygen therapy. J Burn Care Res. 2016;37(1):25-31.

2. Al Kassis S, Savetamal A, Assi R, et al. Characteristics of patients with injury secondary to smoking on home oxygen therapy transferred intubated to a burn center. J Am Coll Surg. 2014;218(6):1182-1186.

3. Tanash HA, Huss F, Ekström M. The risk of burn injury during longterm oxygen therapy: a 17-year longitudinal national study in Sweden. Int J Chron Obstruct Pulmon Dis. 2015;10:2479-2484.

4. Ringbaek TJ. Home oxygen therapy in COPD patients. Results from the Danish Oxygen Register 1994-2000. Dan Med Bull. 2006; 53(3):310-325.

5. Ringbaek T, Lange P, Viskum K. Geographic variation in long-term oxygen therapy in Denmark: factors related to adherence to guidelines for long-term oxygen therapy. Chest. 2001;119(6):1711-1716.

6. Franklin KA, Gustafson T, Ranstam J, Ström K. Survival and future need of long-term oxygen therapy for chronic obstructive pulmonary disease-gender differences. Respir Med. 2007;101(7):1506-1511.

7. Ringbaek TJ, Lange P. The impact of the Danish Oxygen Register on adherence to guidelines for long-term oxygen therapy in COPD patients. Respir Med. 2006;100(2):218-225.

8. Hardinge M, Suntharalingam J, Wilkinson T; British Thoracic Society. Guideline update: The British Thoracic Society Guidelines on home oxygen use in adults. Thorax. 2015;70(6):589-591.

9. World Health Organization. Manual of the International Statistical Classification of Diseases and Health Related Problems. 10th Revision. Vol. 1. Geneva, Switzerland: WHO; 1992.

10. Ludvigsson JF, Andersson E, Ekbom A, et al. External review and validation of the Swedish national inpatient register. BMC Public Health. 2011;11:450.

11. Lynge E, Sandegaard JL, Rebolj M. The Danish National Patient Register. Scand J Public Health. 2011;39(7):30-33.

12. Carlos WG, Baker MS, McPherson KA, Bosslet GT, Sood R, Torke AM. Smoking-related home oxygen burn injuries: continued cause for alarm. Respiration. 2016;91(2):151-155.

13. Vercruysse GA, Ingram WA. A rationale for significant cost savings in patients suffering home oxygen burns: despite many comorbid conditions, only modest care is necessary. J Burn Care Res. 2012;33(6): 268-274.

14. Nocturnal Oxygen Therapy Trial Group. Continuous or nocturnal oxygen therapy in hypoxemic chronic obstructive lung disease: a clinical trial. Ann Intern Med. 1980;93(3):391-398.

15. Medical Research Council Working Party. Long-term home oxygen therapy in chronic hypoxic cor pulmonale complicating chronic bronchitis and emphysema. Lancet. 1981;1(8222):681-686.

16. Litt EJ, Ziesche R, Happak W, Lumenta DB. Burning HOT: revisiting guidelines associated with home oxygen therapy. Int J Burns Trauma. 2012;2(3):167-170. 